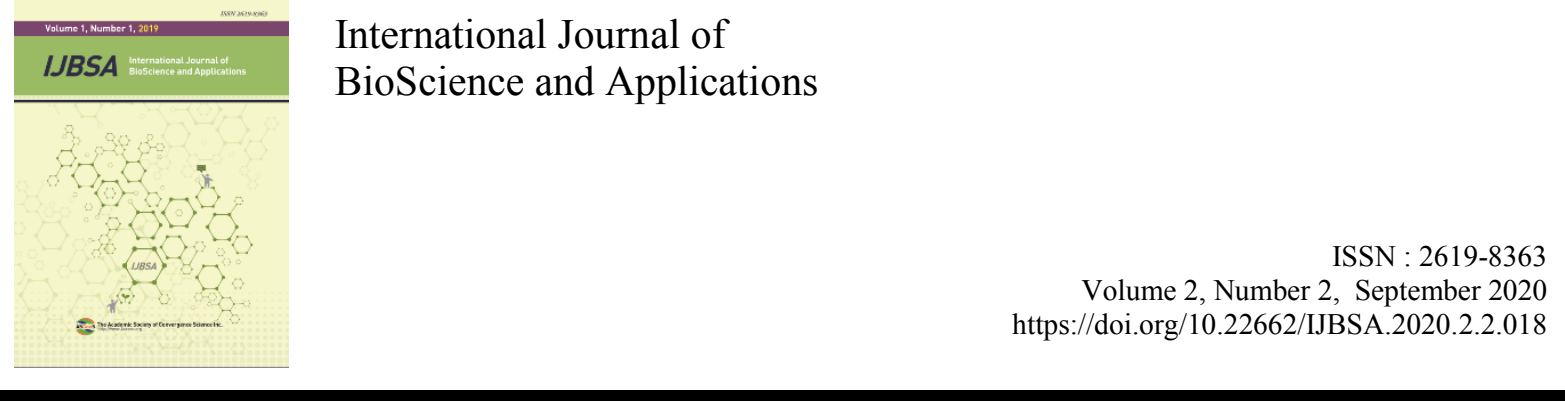

\title{
Development of User Created Content videos for Chinese smartphone users studying in Korea
}

\author{
Hack-Sun Kim ${ }^{1}$, Ja-Sook Kim², Su-Hyun Kim
} Myunghee $\mathrm{Jun}^{4}$, Hye-Kyung Kim${ }^{5}$, and Ja-Ok Kim ${ }^{1 *}$

${ }^{1}$ Department of Nursing, Wonkwang Health Science University, Iksan, Republic of Korea

${ }^{2}$ College of Nursing, Chonnam National University, Gwangju, Republic of Korea

${ }^{3}$ Department of Nursing, Nambu University, Gwangju, Republic of Korea

${ }^{4}$ Department of Nursing, University of Wisconsin-Green Bay, USA

${ }^{5}$ Department of Philosophy, University of Wisconsin-Green Bay, USA

\begin{abstract}
${ }^{1}$
Background/Objectives: The objective of the research was to develop User Created Content (UCC) videos to enhance basic nursing skills for Chinese smartphone users studying in Korea. Methods/Statistical analysis: Working together with Korean students and professors, Chinese international students produced two UCC videos for the inculcation of postoperative nursing skills and instruction in intravenous fluid injections. The videos contained both Korean and Chinese captions. Findings: The process of producing UCC videos by Chinese students was found effective: They participated in the selection of the topics and in the production of the videos. The effectiveness was determined through individual and group interviews. Greater instructional value was also due to the fact that Chinese international students acquired a greater sense of community in working with Korean students and professors in making the videos. Chinese students who participated in the production of the UCC videos could conveniently and effectively learn from their all-too-familiar smartphones.

Improvements/Applications: Production of UCC videos by Chinese international students with Korean students and professors enhances learning basic nursing skills and helps to overcome linguistic and cultural differences. This outcome can present a way to enhance the quality of education and social well-being of international students in Korea.
\end{abstract}

\section{Index Terms}

Alienation, International student, Nursing education, Sense of community, User created content, Video production

\footnotetext{
Corresponding author: Ja Ok Kim

jjaoki-93@wu.ac.kr

- Manuscript received July 20, 2020.

- Revised August 10, 2020 ; Accepted September 2, 2020.

- Date of publication September 30, 2020.

(C) The Academic Society of Convergence Science Inc.

2619-8363 $\odot 2020$ IJBSA. Personal use is permitted, but republication/redistribution requires IJBSA permission.
} 


\section{INTRODUCTION}

Of necessity, Chinese students studying in Korea to obtain a degree in a specialized field utilize the educational resources available in Korea. Such students obviously confront a foreign language, experience a different culture, and grapple with a new environment [1]. In 2012, the Korean government introduced a certification system to attract more international students and efficiently oversee their educational experiences while enrolled in Korean educational institutions [2]. Along with this recent opening of Korean educational institutions to international students, Korean universities have enhanced their international recognition [3]. In particular, a rapid increase in students from China has contributed to the internationalization of Koreanbased education. However, various social problems have emerged. Chinese students experience new different educational and life settings in Korea. As a result, failures to adapt to life in Korea have occurred [4]. This is unfortunate and must be remedied. Since a paramount educational goal in the global era is to produce human beings who can embrace all cultures $[4,5]$, Korean education must adjust accordingly.

Chinese international students experience cultural and medical-education differences that are puzzling to them, and make successful learning more difficult in a Korean educational environment. China has traditionally focused on oriental medicine, and Western nursing methods and practices have not been fully integrated into nursing education in China. Accordingly, international students from China have difficulty understanding technical terms in nursing classes [4,6]. Aadditional instructional aids are thus needed in their nursing training in Korea.

The introduction of e-learning in universities will improve the quality of education and increase the efficiency of teaching and learning [7]. In this study, we develop User Created Content (UCC) videos to be saved in students' smartphone and repeatedly accessed. Smartphones are devices that all students have and can access conveniently in any place and at any time.

The topics covered in the videos were selected by the Chinese international students. They were the topics the students were most interested in during clinical practice. Producing UCC videos on topics students are most interested in obviously enhances learning. In addition, the study explored ways to help Chinese international students overcome cultural estrangement. Creating UCC videos together was instrumental in doing so.

\section{MATERIALS AND METHOD}

\section{A. Study Design}

The objective was to develop two UCC videos by Chinese smartphone using students with Korean students and professors to measure the educational and cultural effectiveness of the UCC video development.

\section{B. Development Process}

1. Participants: Three third-year undergraduate nursing students from China who have had clinical nursing experience. Four Third- and fourth-year Korean undergraduate nursing students who have had clinical nursing experience. Four Korean nursing professors

2. Selection of the subjects of the videos

1) Exploring 20 core skills of Nursing Education Certification Evaluation [8]: Vital sign measurement, oral medication, intramuscular injection, subcutaneous injection, intradermal injection, intravenous fluid infusion, blood transfusion therapy, intermittent gavage, simple urination, primary catheterization, discharge enema, preoperative nursing, postoperative nursing, hospitalization management, protective equipment and the management of waste upon entering the isolation room, the measurement of oxygen saturation and the use of the electrocardiogram monitor, oxygen therapy using nasal cannula, intratracheal aspiration, tracheostomy management, basic CPR and defibrillator application [8].

2) Selection of two basic nursing skills, mastery of which is required for clinical practice: postoperative nursing skills and intravenous fluid injection.

3. Protocol expert check for intravenous fluid infusion and post-operative nursing [8-10]

4. Translating the Basic Nursing Skills Protocol from Korean into Chinese

1) Translation of intravenous infusion protocol into Chinese by three Chinese international students

2) Translation of post-operative nursing protocol into Chinese by three Chinese international students

5. Video of basic nursing skills using smartphones by Korean students

1) Video, by smartphone, of intravenous fluid infusion by Chinese and Korean students; setting: Basic Nursing Practice Lab

2) Video, by smartphone, of post-operative nursing care by Chinese and Korean students; setting: Basic Nursing Practice Lab

6. Analysis of recorded videos by nursing professors [11-14] 
1) Protocol and video analysis

2) Secondary comments and suggestions for video revisions

7. Revision of the videos

8. Chinese experts' check of the translation of the protocols for nursing skills

9. Chinese synthesis

1) Chinese subtitles added; video experts and Chinese student team consulted

2) Chinese audio added; video experts and Chinese student team consulted

10. Post-editing video assessment: visual and auditory quality, fidelity to protocols, educational effectiveness [8-14].

1) Assessment by a nursing specialist of video quality and fidelity to protocols

2) Assessment by Korean students of video quality and educational effectiveness

3) Assessment, by Chinese students, of video quality and educational effectiveness

11. Completion of research project: two UCC videos on basic nursing skills by Chinese smartphone users studying in Korea

\section{RESULTS AND DISCUSSION}

The UCC video production was found to be an effective learning tool. The benefits of developing UCC videos have been confirmed in scholarly articles [15]. The content of the videos was enriched, and greater educational benefits reported, by Chinese students working in cooperation with Korean students and professors in the production of the UCC video.

The addition of Chinese caption into the videos, making them truly bilingual, ensured that they were more user-friendly than Korean-only videos. The Chinese international students were much more receptive to instruction in basic nursing skills in their native tongue. They also showed greater progress in mastering such skills after instruction in their native tongue. The language barrier is one of the most serious obstacles for Chinese students. Bilingual videos can be successful instruments in facilitating learning in Korean educational institutions.

Educational effectiveness was enhanced by active cooperation with Korean students. The success achieved was primarily due to Chinese students' planning, making, and assessing videos with Korean students. This engagement increased the quality of the video content. It also strengthened the sense of community with Korean students and professors.

Engagement was multi-faceted and rewarding. Using their smartphones, Chinese and Korean students recorded videos of basic nursing skills. They then edited the videos to reflect nursing protocols as accurately and perspicuously as possible. Chinese international students translated the in-Korean protocols into Chinese. The translated protocols were reviewed by Chinese-language experts. Chinese students then participated in preparing subtitles and in effecting dubbing. The interactions of Chinese and Korean students in diverse but integrated activities reinforced a sense of community in the nursing department. Such a sense of community eased anxieties due to cultural, linguistic, and environmental displacement, and helped to facilitate learning.

Active participation by both Chinese and Korean students is particularly important to stress. Learning by doing is hardly a new idea, but its advantages over purely passive instruction has been well argued by the famous philosopher of education John Dewey [16]. In addition to easing cultural differences, theory, practice, and self-worth are all conveyed by hands-on participation.

The use of smartphones was particularly effective. Smartphone utilization fits the learning style of the younger generation, and thus facilitates efficient learning. Shorter exposure times in learning experiences also fits with the learning style of the younger generation and is very amenable to smartphone utilization. Evidence for this includes a case study on the use of e-learning contents for engineering students. The study showed that it was more efficient to create and provide contents shorter than 25 minutes [17]. The two videos created for use by Chinese students were approximately 10 minutes each (Figure 1, 2).

Proposed extensions of this study include testing the validity of the findings by varying a number of factors, for example, subject matter (e.g., more advanced nursing skills), nationality (e.g., Mongolian or Vietnamese natives), size and relative composition of foreign and domestic student bodies (e.g., 10 and 10 domestic students, or 10 foreign students and 5 domestic students), and length of videos (e.g., longer and shorter than 10 minutes).

Example A. Intravenous Infusion Injection

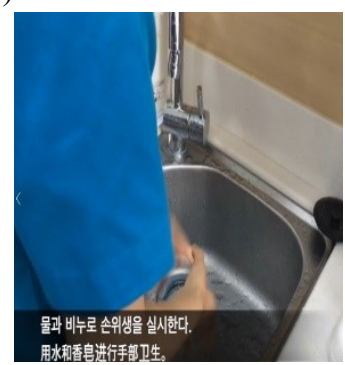



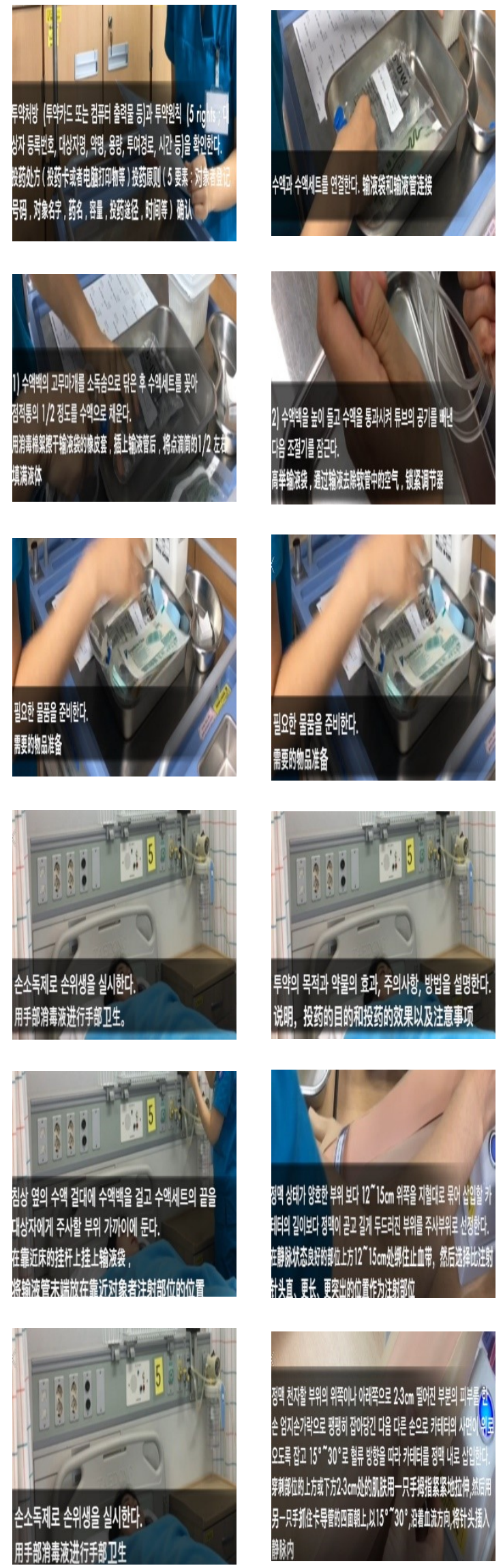
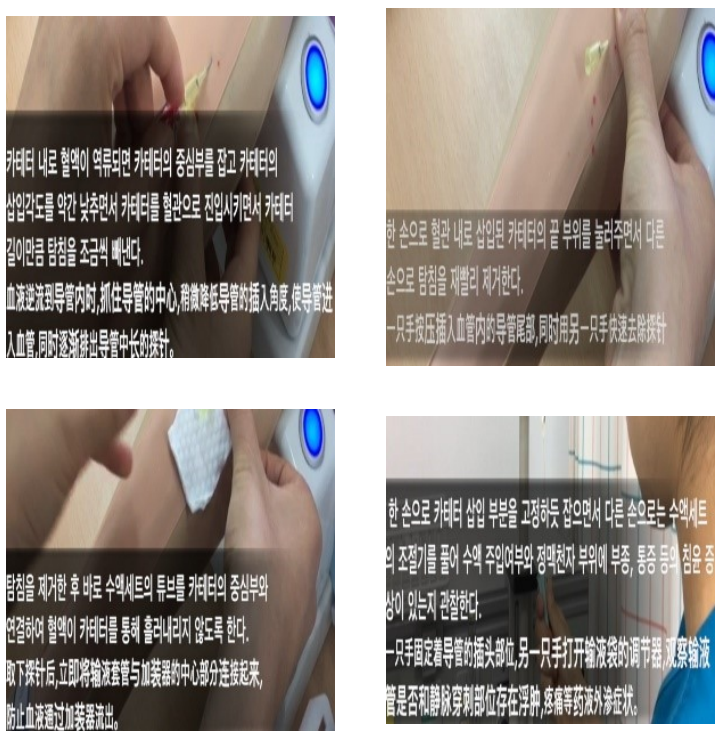
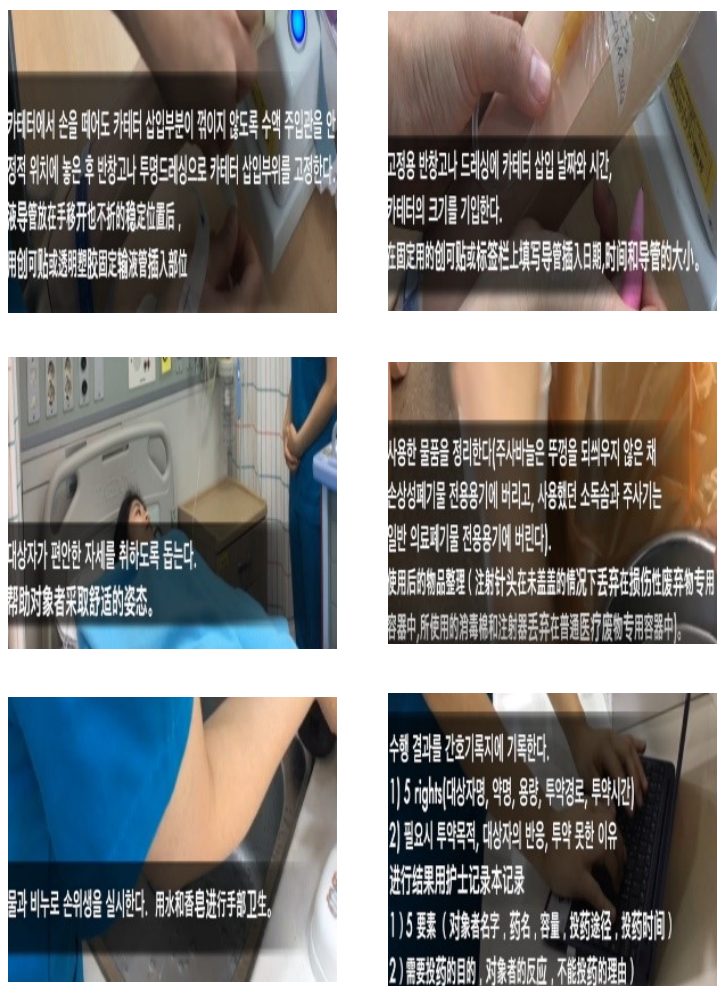

Fig. 1. The example A of intravenous infusion injection video contents

Example B.

Postoperative Nursing

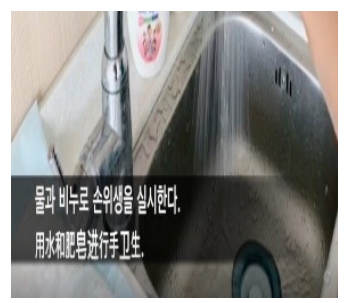



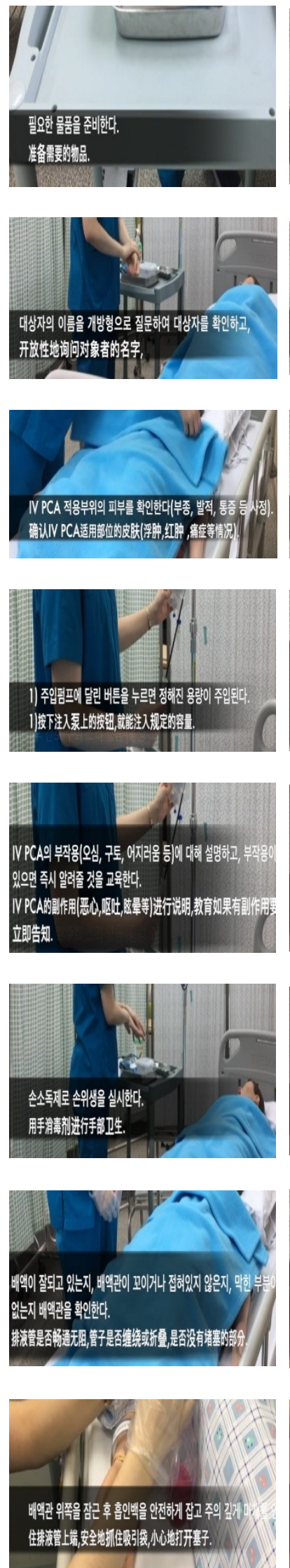
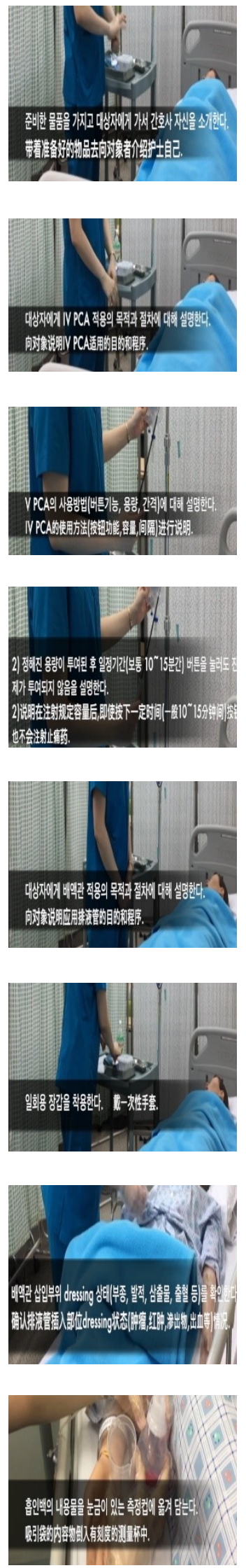
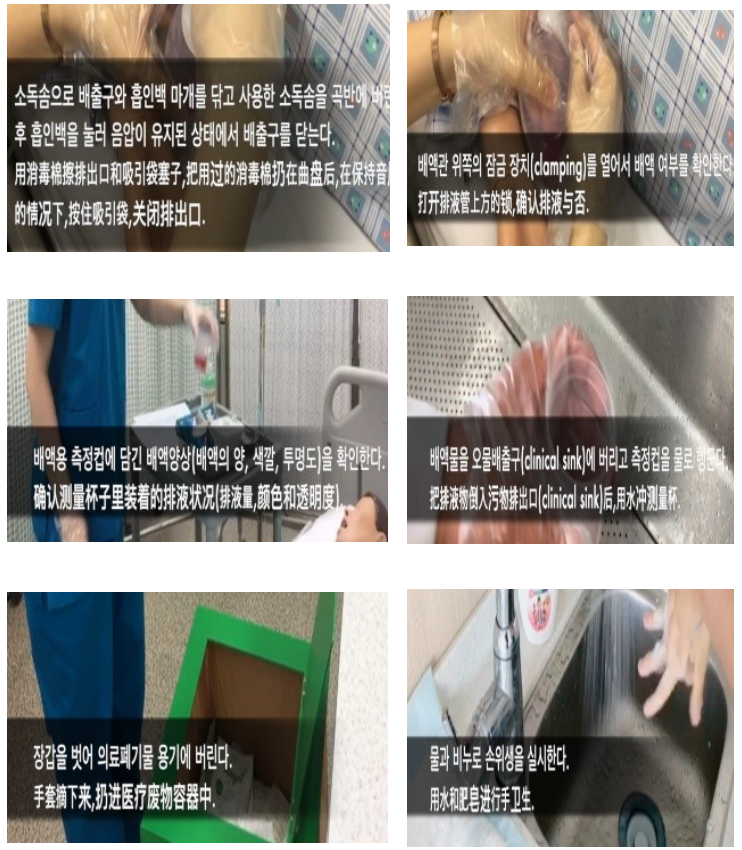

\section{REFERENCES}

[1] Philip, H (2015). The MSA: An Instrument for Measuring Motivation to Study Abroad, Interdisciplinary, Journal of Study Abroad, Vol XXVI, Fall.

[2] Zhang, S. X., \& Li L. Z (2019). A Study on the Relationship between the Self-Growth Motivation and the Life Satisfaction of Overseas Chinese Students in Korea : Focused on the Mediation Effect of Job Search Efficacy, The Journal of Business Education, 33(5), 125. DOI : $10.34274 /$ krabe.2019.33.5.001

[3] Chang, S. B., \& Yang, H. S. (2012). Chinese Students' Learning Effect Impact on University Life, Journal of Digital Convergence, 10(11), 67-80.

[4] Shin, S. H., \& Yu, M. M. (2014). A Qualitative Study on the Acculturation Experiences of the Chinese Students at a South Korean University, Journal of Holistic Convergence Education, 18(2), 45-69.

[5] Wang, K. T., Heppner, P. P., Fu, C. \& Zhao, R. (2012). Profiles of acculturative adjustment patterns among Chinese international students. Journal of Counseling Psychology 59(3), 424-436.

[6] Guofang, W. (2001). The learning experience of Chinese students in American universities: A crosscultural perspective. College Student Journal, 35(1), $28-45$.

[7] Lee, H. J., Hong, Y. I. \& Son, J. Y. (2007). Effects of online feedback types among students on learning outcome in a blended e-Learning environment, The Journal of Educational Information and Media, 13(3), 131-157.

[8] Korea Institute of Nursing Education Evaluation (2017). Nursing Education Certification Evaluation Core Basic Nursing Skills Evaluation Item Protocol(4.1 version). Korea Institute of Nursing Education Evaluation.

[9] Yang, S. H. et al. (2020). Fundamental Nursing I. Seoul:Hyunmoonsa. 
[10] Yang, S. H. et al. (2020). Fundamental Nursing II. Seoul:Hyunmoonsa.

[11] Kim, H. S. \& Kim, J. S. (2019). Understanding Adult Nursing for Chinese Students. Seoul:Eunhaksa.

[12] Kim, J. S., Ji, H. L., \& Kim, J. O. (2020). Core Fundamental Nursing guidebook I. Seoul:Eunhaksa.

[13] Kim, J. S., Ji, H. L., \& Kim, J. O. (2020). Core Fundamental Nursing guidebook II. Seoul:Eunhaksa.

[14] Yang, S. H. et al.(2020). Core Basic Nursing Skills. Seoul: Hyunmoonsa.

[15] Greene, H. (2014). Learning through student created, content videos. International Journal of Arts \& Science, 7(02), 469-478

[16] Dewey, J. (1938). Experience and education. N.Y: Touchstone.

[17] Park, H. K. (2017). A Case Study on Utilization of elearning Contents for Engineering Education, Journal of Practical Engineering Education, 9(2), 133-137. 